\title{
USE OF FILTERED COMBUSTION LIGHT AND BACKLIT HIGH-SPEED IMAGES IN COMBUSTION STABILITY STUDIES
}

\author{
B. Pomeroy, M. Wierman, and W. E. Anderson \\ Purdue University, School of Aeronautics \& Astronautics \\ 701 W. Stadium Ave., West Lafayette, IN 47907-2045, USA
}

\begin{abstract}
The measurement of the heat release is a key part of characterizing the combustion instability, but it is extremely difficult to directly measure in a rocket combustion chamber due to high temperatures and pressures, as well as the complexity of the turbulent reacting flowfield, which can often have more than one phase. Measuring the light emission from excited species during a combustion is a nonintrusive method to approximate a global heat release in combustion chambers. $\mathrm{CH}^{*}$ and $\mathrm{OH}^{*}$ are the most often measured species. This paper outlines methods of using a filtered combustion light to obtain a better understanding of the physical mechanisms active in the combustion instability, and to provide partial validation data for predictive models of the combustion instability. Methods that are discussed include Rayleigh index, phase-angle plots, a proper orthogonal decomposition (POD), and a simultaneous imaging of combustion light and backlit flow structures. The methods are applied to an experiment that studies the effects of imposed transverse oscillations on a gas-centered, swirl-coaxial injector element.
\end{abstract}

\section{INTRODUCTION}

A high frequency combustion instability can be detrimental in a rocket engine when the unsteady heat released from the combustion process is coupled with resonant acoustic pressure oscillations in the chamber. Rocket engines are more prone to stability problems because the extreme heat release has a very tight spatial coupling to the chamber acoustics, and the combustor is mostly closed with relatively little means for acoustic losses. Many rocket engines have suffered from combustion stability problems during development, and solving the problem during testing can drastically increase the cost and significantly delay the process. The typical strategy to correct the instability is to increase the overall damping

This is an Open Access article distributed under the terms of the Creative Commons Attribution License 2.0, which permits unrestricted use, distribution, and reproduction in any medium, provided the original work is properly cited. 
of the system or reduce the coupling between the unsteady combustion and the pressure oscillations. However, due to the problem's complexity, this is not at all straightforward, and finding some means for accurately predicting the instability prior to a full-scale development is of great value. Simulations of rocket engine instabilities via computations are promising, but they are still very much in development [1].

An accurate determination of the combustion response, a measure of how the reacting flow field responds to oscillations in the pressure and the velocity, would be a major improvement in the combustion stability prediction. Theoretically, combustion response can be determined experimentally or from high-fidelity simulations, and can be used in stability predictions [1]. High-speed quantitative measurements of chemical species would be ideal, but have not yet been applied to the unstable rocket environment. The actual measurement of the heat release in a practical combustion device is a matter of research, and even indirect measurements of combustion response in rocket engines are quite difficult due to extremely high heating rates and the high-pressure environment, so indirect measurements are used instead.

This paper describes how high-speed imaging is used to measure the flow structure and the flame response in a model rocket combustor that produces a flowfield that represents a transverse combustion instability. Using unstable injector elements near the end walls of a rectangular combustor provides the oscillatory flowfield that has a first mode frequency of about $2000 \mathrm{~Hz}$. An injector element of interest is placed in the center of the chamber, where the velocity oscillations of the first mode are greatest. Opposing windows near the study injector element allow optical access to the local fluid mechanics. Backlit images are taken to study the dynamic flow structure of the propellant flow from the injector, and the chemiluminescence from excited species occurring during the combustion process is used to approximate the location and the oscillatory response of the flame. Emitting species that have been used to measure heat release include $\mathrm{CH}^{*}, \mathrm{OH}^{*}, \mathrm{CO}_{2}^{*}$ and $\mathrm{C}^{*}[2,3]$. $\mathrm{CH}^{*}$ is used often in hydrocarbonfueled flames since it is a shorter-lived species and its wavelength is in the visible regime allowing for simpler optics $[2,3]$. The emission of $\mathrm{CH}^{*}$ occurs around $430 \mathrm{~nm}$ whereas $\mathrm{OH}^{*}$ emission is centered in the UV region of $307 \mathrm{~nm}$.

The chemiluminescence measurement is a line of sight technique used to determine the total heat release emitted by a flame in the region of interest [4-11]. Balachandran et al. studied both $\mathrm{OH}^{*}$ and $\mathrm{CH}^{*}$ simultaneously in a series of experiments and determined that both were in good agreement with each other and are a good indicator of global heat release [9]. The study found that the $\mathrm{OH}^{*}$ and $\mathrm{CH}^{*}$ global measurements correlated with the global heat release measurements, and the magnitude and the phase of the global $\mathrm{OH}^{*}$ and $\mathrm{CH}^{*}$ were in good agreement. A few studies have shown that $\mathrm{OH}^{*}$ and $\mathrm{CH}^{*}$ chemiluminescence does not directly measure the exact location of heat release especially in high strain rate environments $[4,5,10]$. Recently, the groups in France, Ger- 
many, and the USA have been using $\mathrm{CH}^{*}$ and $\mathrm{OH}^{*}$ chemiluminescence to study the instability in model rocket combustors [11-13]. Although the measurements do not provide a direct and quantitative measure of heat release, they still can be used to indicate the location and global response, and are a valuable diagnostic that can provide understanding and means for verifying computer models.

This paper presents a series of data reduction methods used to study how reacting flows from rocket-type injector elements respond to imposed flow oscillations. First, an overview of the experiment is provided, including a brief description on how the flow oscillations are produced. Then each reduction method is discussed in turn. The reduction methods include analyzing the intensity signals to determine locations of an average combustion, locations of the maximum oscillation, and regions of driving and damping as well as how the velocity interacts with the flame both in a general fashion and for the first few excited modes.

\section{EXPERIMENTAL SETUP}

The chamber used in the present study (Fig. 1a) has seven linear gas-centered swirl coaxial injectors which were derived from oxidizer-rich staged-combustion engines. The elements near the end walls of the combustor are unstable, and drive the transverse instabilities. The injector element in the middle of the chamber is the "study element." Decomposed $90 \%$ hydrogen peroxide flows

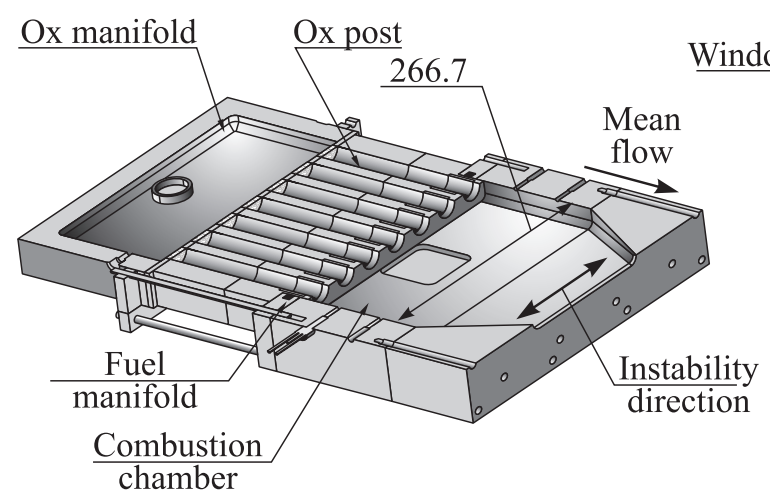

(a)

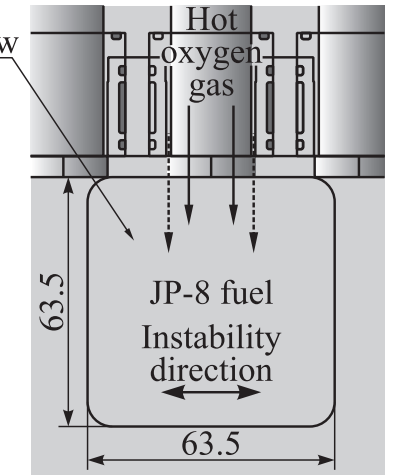

(b)

Figure 1 (a) A cutaway view showing the chamber's oxidizer manifold, oxidizer posts, fuel manifolds, the chamber with the window in the center just downstream of the injector face and the converging nozzle; and $(b)$ detailed view of the gas-centered swirl coaxial injector with the window. Dimensions are in millimeters 
through all seven oxidizer posts. To isolate the effects of the oscillating flow, fuel (JP-8) flows only through the outer two elements on each side of the chamber and the study element in the center of the chamber, so that oxidizer jets on both sides isolate the study element. A more detailed view of the center study element is shown in Fig. 1b. A detailed description of the combustion chamber can be found in [13].

A Vision Research Phantom V7.1 high-speed camera was set up in a protective box with an optical filter (Schott BG39) fully covering the front opening. This filter allowed light in the range between $370 \mathrm{~nm}$ and $580 \mathrm{~nm}$ to reach the camera at a greater than $80 \%$ transmittance; other filters with a finer resolution have also been used. The camera is synchronized with high frequency pressure measurements at $100 \mathrm{kHz}$ to provide correlations between the image and the local unsteady pressure field. The velocity field can be calculated from the measured pressure field.

The measured mode shapes have been studied to verify that the transverse oscillation is a standing wave and fits a theoretical acoustic wave. With the standing wave assumption, the pressure is estimated across the chamber by using high frequency pressure transducers to determine a pressure coefficient. There are six high frequency transducers located around the window and one transducer on the side of the chamber wall. These seven transducers and their respective locations are used to fit a coefficient for a theoretical cosine pressure wave for each instant of time. This coefficient and the theoretical cosine wave are used to build the pressure across the window for each frame.

The velocity in the chamber is complicated due to the heterogeneous regions of hot combustion products and warm decomposed hydrogen peroxide. Thus the acoustic velocity will vary through each of these regions, and their exact boundaries are not well defined due to the oscillation in the chamber. For this study the flowfield was approximated to be homogeneous and velocity was determined from the measured pressure gradient.

The camera's settings vary slightly for each experiment, but its frame rate is set depending on the instability frequency. The highest resolution is used for the required frame rate. Two different setups are used depending on the ultimate goal of the study. To determine the location, frequency, relative intensity, and the phase of the heat release with respect to the pressure wave, only the camera with the optical filter is used as shown in Fig. 2. Here, the windows for the light path are located at the injector face in the center of the combustor. The filtered combustion light and the location were recorded, as well as the relative heat release and frequency.

The second setup (Fig. 3) includes an additional synchronized camera that is used to simultaneously record backlit images, providing some information of the relationship between the heat release and the visible flow structures, in this case the flow of liquid propellants through the chamber. These data allow for a correlation between the temporal and spatial response of the emission sig- 


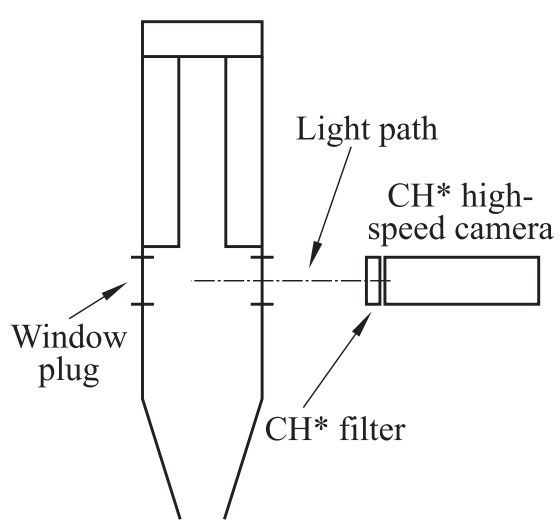

Figure 2 Chemiluminescence imaging schematic. The high-speed camera has a filter for the $\mathrm{CH}^{*}$ imaging [14]

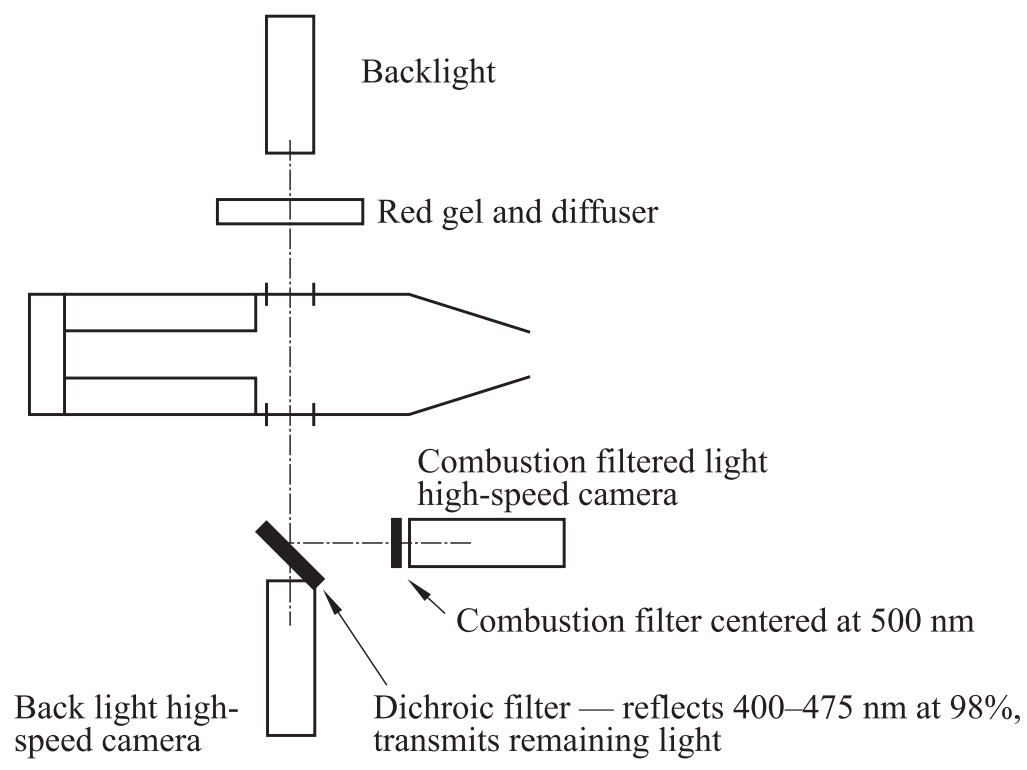

Figure 3 Simultaneous backlight shadow images are taken at the same time as $\mathrm{CH}^{*}$ combustion images through the use of a dichroic filter [14]

nal, movement of the fuel jets and spray, and the velocity and pressure waves. Presumably, this combined information allows the development of a mechanistic response model through a more complete understanding of the combustion dynamics. 
The two-camera setup has the extra complexity of three synchronized systems, but provides more information than the filtered light alone. The synchronized system uses a dichroic filter mirror that splits the light coming from the chamber. The current dichroic mirror setup is used with the camera that is recording the backlit images. It reflects the light from 400-475 nm (greater than $98 \%$ reflection) to the camera recording the filtered combustion light while transmitting the remaining backlight at greater than $90 \%$. A specific red gel filter (transmits light greater than $600 \mathrm{~nm}$ ) is used over the backlight in order to only transmit red wavelength light above the reflected wavelength. This assures that the camera will detect the filtered combustion light.

The images are analyzed with methods developed in-house. Raw video files are used to reduce the loss of information in the images. A large number of frames are selected which span numerous pressure oscillations. The information extracted from the plots is then compared using a variety of methods discussed below.

\section{METHODS}

The reduction methods should provide understanding of the combustion dynamics and means for model validation. No single image gives enough information to gain this understanding into combustion dynamics, so all images must be analyzed together. Similarly, no single method provides all the necessary information. An important area for future work is to investigate how the methods can be systematically analyzed to provide understanding and the validation.

Contour plots of combustion light intensity show where the combustion is occurring in the chamber. Filtering these images around a certain frequency can show the spatial response to specific modes. An analysis of the temporal correlation between the light emission and the local unsteady flowfield parameters yields the phase difference between the intensity signal (representing the heat release) and the oscillating flowfield. These plots are useful when used along with the plots of the Rayleigh index to indicate regions of driving or damping. From the filtered intensity plots and the measured pressure field, a combustion response can be determined, at least in principle.

The representative plot shown in Fig. $4 a$ is a contour plot of the root mean square (RMS) of the total light intensity. It shows a time-averaged approximation of the locations where the greatest fluctuations of the heat release occur. The image gives an insight into the location and relative intensity of the heat release. On the other hand, Fig. $4 b$ shows a spatially resolved RMS of the filtered light oscillations, calculated by determining the RMS of a single pixel's time series of the filtered light. Figure $4 b$ was filtered for the primary $1 \mathrm{~W}$ mode of $2032 \mathrm{~Hz}$ and shows distinct regions of the flame (indicated by the red color) 


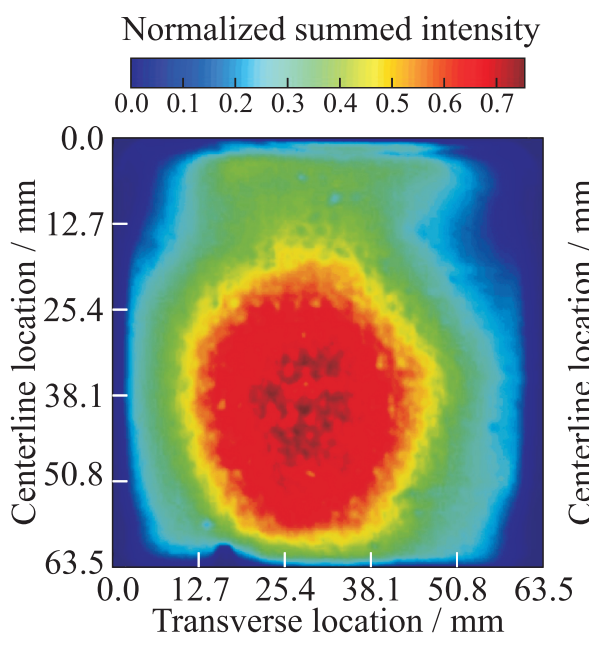

(a)

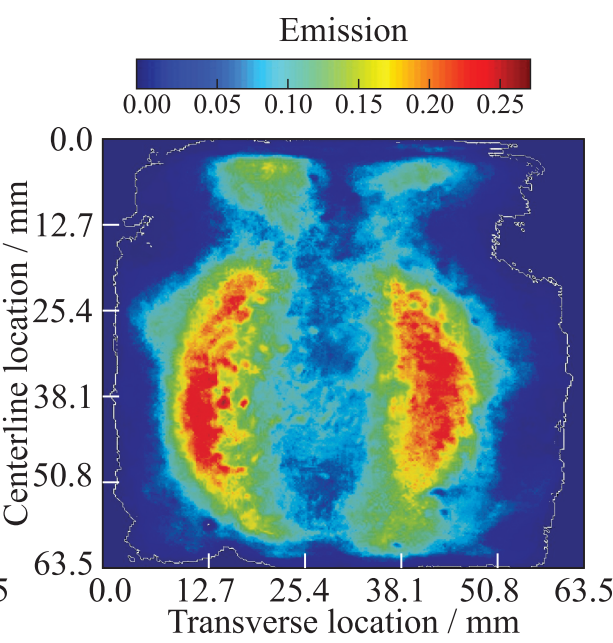

(b)

Figure 4 (a) Normalized summed intensity from a series of images (taken through the window shown in Fig. 1b) and (b) an RMS image showing the spatial regions where the emission oscillates most (for $2032 \mathrm{~Hz}$ ). The injector flow is from the top to the bottom.

most susceptible to $1 \mathrm{~W}$ mode oscillations. Since the study element is located at the $1 \mathrm{~W}$ velocity antinode, it can be reasonably assumed that the regions of the response shown in Fig. $4 b$ are due to transverse velocity oscillations.

The Rayleigh index

$$
\text { Rayleigh index }=\frac{1}{\bar{p} \bar{q} \Delta t} \int_{0}^{t} p^{\prime} q^{\prime} d t
$$

is commonly used to describe regions of driving and damping. Negative and positive values of the Rayleigh index indicate damping and driving, respectively. In the Rayleigh index plot of Fig. $5 a$, the local instantaneous pressure is calculated from discrete pressure measurements and the instantaneous chemiluminescence. Increasing magnitude of the response is denoted by darker shades of red (positive) and blue (negative). This Rayleigh index plot uses the oscillating pressure signal filtered around the $1 \mathrm{~W}$ mode frequency of $2032 \mathrm{~Hz}$, so $p^{\prime}$ near the centerline should be close to zero at all times. Note the similarity between the Rayleigh index plot and the plot of intensity variation shown in Fig. $4 b$.

Although it is a useful indicator, the Rayleigh index lacks key information on the phase angle between the combustion light emission and the associated pressure. Also, since it is a product, the Rayleigh index does not indicate the 


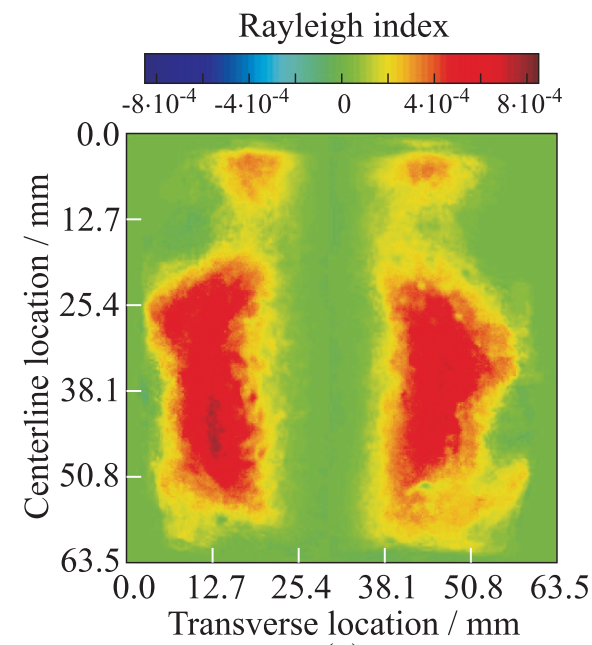

(a)

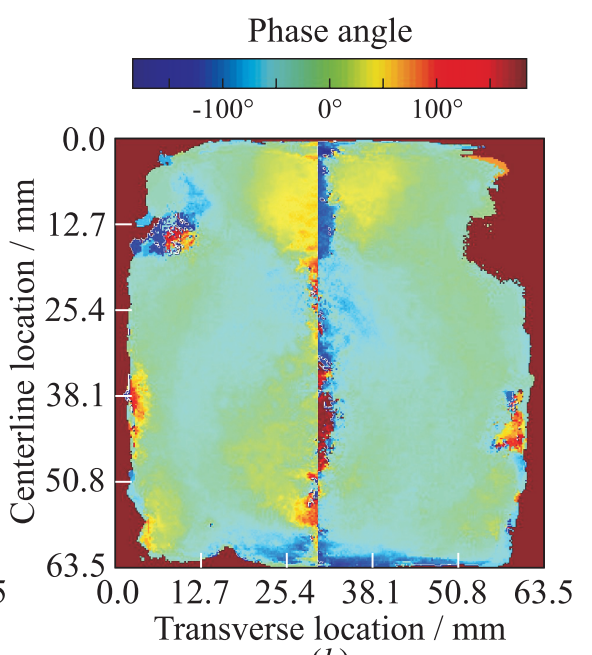

(b)

Figure 5 (a) Rayleigh index plots show the area of greatest driving and damping within the combustion chamber. The dark red areas show portions of the flow that respond with the greatest magnitude driving the instability. It can be seen that the reacting flow from the coaxial injector shown in Fig. 1 responds most significantly to $1 \mathrm{~W}$ transverse waves in the mixing zones outside the inner jet core; and $(b)$ phase for the pressure and light emission. Injector flow is from the top to the bottom; $f=2032 \mathrm{~Hz}$.

sign of $p^{\prime}$ or $q^{\prime}$. Therefore, it is not possible to determine whether a driving condition results from the coincident expansion and a deficit of heat release, or from the coincident compression and the surplus of the heat release.

Knowing the phase of the heat release with respect to the pressure and velocity waves is important to understand how the oscillating pressure and velocity fields affect the unsteady heat release. Generally, the peak pressure is used as an arbitrary reference. To produce the phase plots, such as the one in Fig. 5b, the pressure measured in the chamber is extrapolated across the window region and a phase angle between the pressure and the light intensity can be determined. The phase is presented as $\pm 180^{\circ}$. A positive phase angle indicates that the pressure leads the combustion wave, and a negative value indicates that the pressure lags the combustion. This can be very useful, providing, for example, information on whether a spike in the heat release occurs near the onset of a compression wave, or after the compression wave has passed. Care must be taken in this calculation, since the filtering technique can affect the time at which the pressure is a peak.

When calculating the phase angle two different methods have been used giving similar results. The first option used a code developed in-house to determine 
neighboring peaks in a signal. Next, the code calculated the phase angle in a second signal with respect to its peak location between the two peaks. This method is easy and can quickly produce a 0 to 360 degree plot as a function of time. The second option used a Matlab function to determine the cross correlation between two signals. This function calculates the best overlap between two signals and returns the angle of shift for the period of time. This function is easy to implement since it is built-in to Matlab, but will not calculate the phase angle through the time.

In developing the Rayleigh index plots, methods for normalizing the pressure and light intensity peak-to-peak values were studied. The average chamber pressure is used as well as the average light intensity over the entire window for the duration of the light images in question. Using the average intensity over the entire window was compared to an average value of the intensity from individual pixels. The results showed that the trends were similar, but the peak values were not as high when using individual pixels. Also, it was found that normalizing with the average intensity for each pixel exhibited greater sensitivity when the intensity signal was low.

It is also possible to generate Rayleigh-type plots based on the velocity, where the velocity calculated from the measured pressure gradient is substituted for the pressure as

$$
\text { Velocity Response }=\frac{1}{\overline{u q} \Delta t} \int_{0}^{t} u^{\prime} q^{\prime} d t .
$$

When viewed in conjunction with the velocity-combustion plots, the Rayleigh plots can help determine which portion of the combustion is occurring during the direction of the velocity wave.

The representative velocity-based plot in Fig. $6 a$ shows a response similar to the pressure-based plots above. In this plot, the positive velocity travels from the left to the right. A high response is noted by either dark areas of red or blue. The red portion shows a response for the rightward traveling wave multiplied by a value of high combustion intensity, while the blue portion shows the area with a leftward traveling wave multiplied by a value of high combustion intensity. These plots can be used to determine which direction the wave is traveling for a combustion response. The phase of the velocity-combustion is shown in Fig. $6 b$. Knowledge of the phase indicates the time in the velocity cycle at which the combustion is occurring; a positive value denotes that the velocity leads the chemiluminescence signal and a negative value indicates the velocity lags light emission.

Proper orthogonal decomposition of the filtered combustion light images can be used to extract a spatial and temporal response based on the energy of the response. One advantage of the POD is its ability to indicate a spectro-spatial response without prior knowledge of the frequency of interest, which can be 


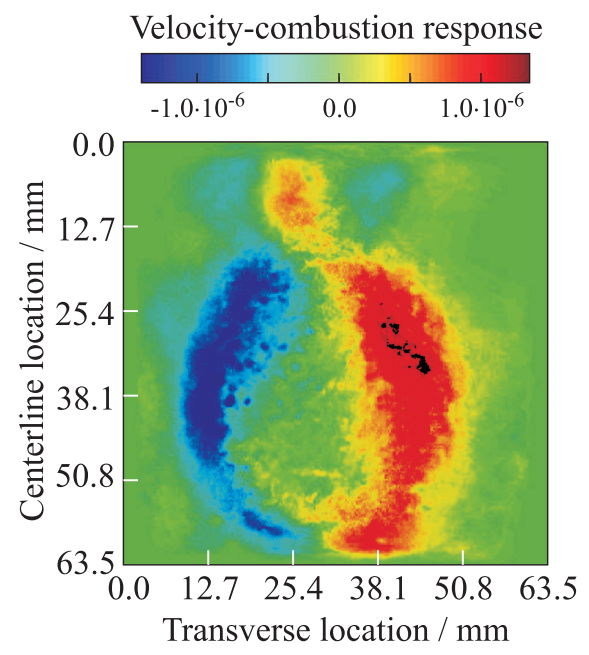

(a)

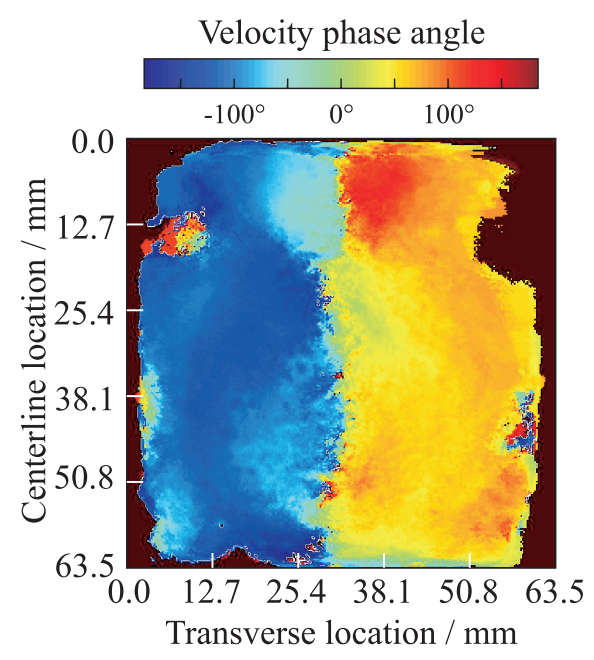

(b)

Figure 6 (a) The velocity-based plot is calculated similar to the Rayleigh index, but with the velocity instead of the pressure. A positive traveling velocity wave moves from the left to the right; and $(b)$ the phase angle for the velocity-based plot. Frequency $2032 \mathrm{~Hz}$.

particularly useful when forcing is low. Figure 7 shows the location and the relative strength of the first three spatial energy levels corresponding to the $1 \mathrm{~W}$ and $2 \mathrm{~W}$ frequency. The first two energy levels (see Figs. $7 a$ and $7 b$ ) are nearly mirror images indicating a separate response on each side of the injector. When animated, the alternating response can be seen showing a response in each location as the wave travels past the injector. Figure $7 c$ shows the response of the $2 \mathrm{~W}$ mode.

A POD also returns a temporal signal, which can be analyzed through a power spectral density for frequency content. The frequency of the temporal signal can be used to determine the frequency of the POD mode. Figure 8 shows the first three temporal responses for the respective spatial images in Fig. 7. Figures $8 a$ and $8 b$ show a strong $1 \mathrm{~W}$ response at $2032 \mathrm{~Hz}$, at the same location as measured by the pressure transducers. Likewise, Fig. $8 c$ shows the $2 \mathrm{~W}$ response at $4065 \mathrm{~Hz}$. The temporal and spatial signal can be used to rebuild the original video signal. Work is being conducted to take the temporal and spatial signal from the POD to develop a response function.

Finally, simultaneous images of backlit and chemiluminescence can provide information regarding the relationship between flow structures and the heat release. In Fig. 9, the injector flow is from top to bottom and a compression wave traverses the chamber from the left to the right. The individual filtered com- 


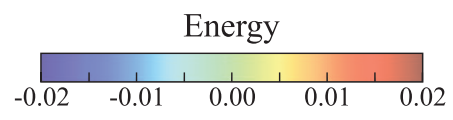

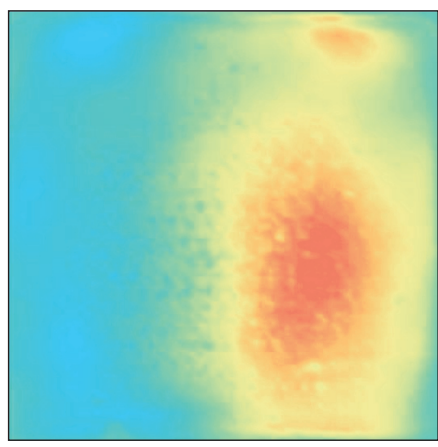

(a)

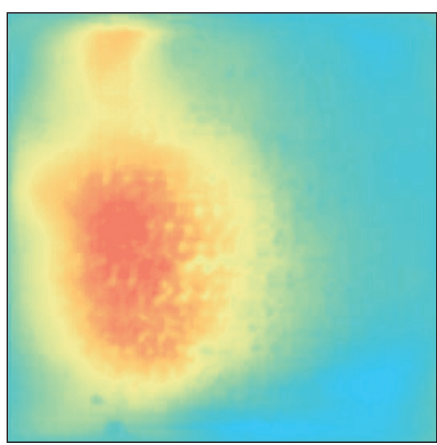

(b)

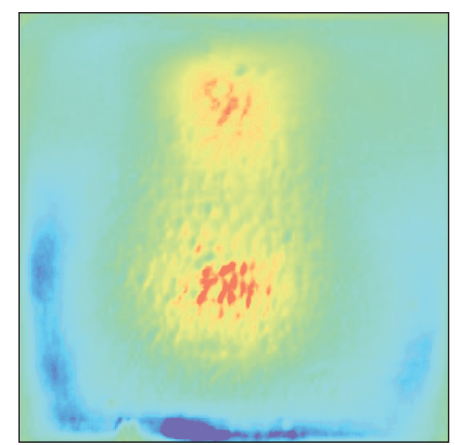

(c)

Figure 7 Proper orthogonal decomposition of the high-speed video reveals two separate but distinct locations of combustion for the $1 \mathrm{~W}$ frequency: $(a)$ the strongest POD energy response is located on the right side of the video image and $(b)$ a slightly lower, but strong response is located on the left side of the images; and $(c)$ the third level of energy shows a part of the $2 \mathrm{~W}$ frequency response. Injector flow is from the top to the bottom.

bustion images can be analyzed using the above four techniques, but having the additional knowledge of the liquid propellant trajectory can help develop a better understanding of the root cause of the unsteady response. Figure 9 shows an image taken in a highly unstable chamber with peak-to-peak oscillations nearly $100 \%$ of the chamber pressure. The backlit image produces the main image in which the center of the light intensity is overlaid from the combustion-filtered image. More information on how this is calculated can be found in [14]. The green line represents the center of intensity calculated for each row and the yel- 


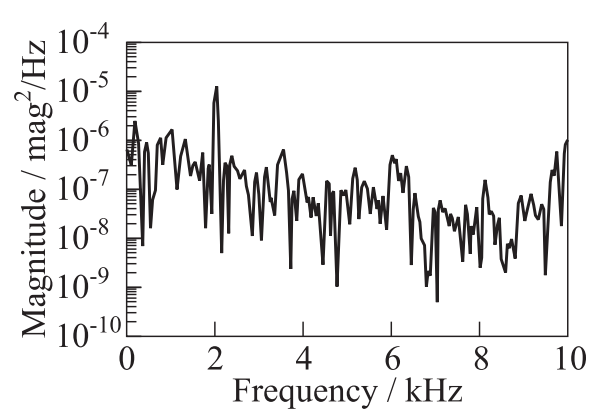

(a)

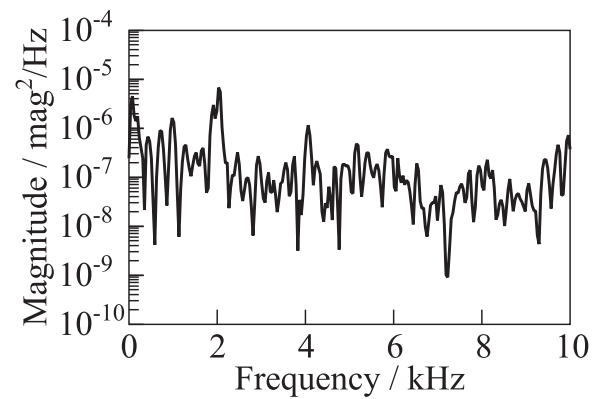

(b)

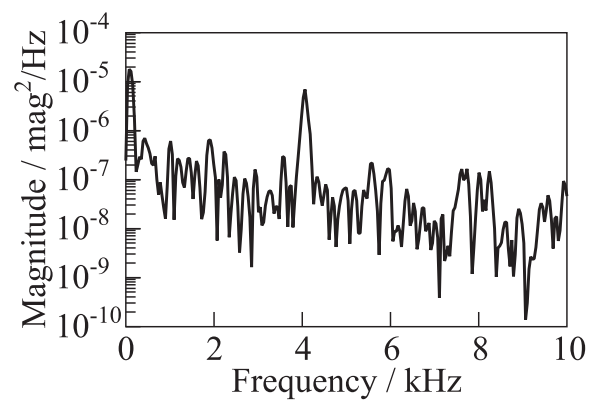

(c)

Figure $8(a)$ and $(b)$ A power spectral density of the temporal signal shows distinct peaks at the $1 \mathrm{~W}$ frequency of $2032 \mathrm{~Hz}$ and (c) shows the $2 \mathrm{~W}$ frequency of $4065 \mathrm{~Hz}$. The figures respectively show the temporal response for the respective spatial response in Fig. 7

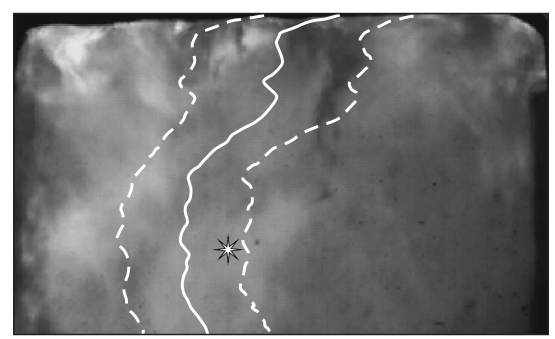

Figure 9 Simultaneous images record both the backlit spray structures and the filtered light from combustion. Here, the filtered light is represented by the center of intensity indicated by the solid line and a plus/minus one standard deviation is represented by the dashed lines. The star represents the center of intensity for the entire view field 
low line is the plus or minus one standard deviation. The red dot represents the center of intensity in both the horizontal and vertical direction for the entire image. In the lower portion of the image it can be seen that the majority of the combustion is biased towards the left side of the image. While work is currently being conducted on how to improve the quality of the images, it can be seen how the velocity wave can move the propellants around in the chamber and cause the fuel to mix together at locations other than directly downstream of the injector.

\section{CONCLUDING REMARKS}

To understand the complexities of the high frequency combustion stability problem, multiple measurements and data analysis techniques are necessary, along with high-fidelity simulations. In conjunction with sets of high frequency pressure measurements to indicate pressure and velocity mode shapes, the use of high-speed chemiluminescence measurements allows an analysis of the spatial and temporal characteristics of the unsteady heat release. It is also important to know how much heat is released, and the phase with respect to the pressure or the velocity wave. Backlit images of flow structures show how the liquid propellant is distributed through the chamber. Adding this knowledge to the temporal and spatial chemiluminescence allows for a more complete comparison between experimental and numerical combustion results. As the comparison between the backlit and chemiluminescence images progresses, numerical models can be improved as the understanding of the physics in the combustion chamber grows. Knowing how the injector responds to pressure and velocity waves, response functions can be developed to be used in improved engineering level stability design analysis tools.

\section{ACKNOWLEDGMENTS}

The authors would like to thank the NDSEG Fellowship, State of Indiana, INSpace LLC, and the NASA Constellation University Institute Project for their continued support of this research.

\section{REFERENCES}

1. Smith, R., M. Ellis, G. Xia, V. Sankaran, W. Anderson, and C. Merkle. 2008. Computational investigation of acoustics and instabilities in a longitudinal mode rocket combustor. AIAA J. 46(11):2659-73. 
2. Walsh, K. T., M. B. Long, M. A. Tanoff, and M. D. Smooke. 1998. Experimental and computational study of $\mathrm{HC}, \mathrm{CH}^{*}$, and $\mathrm{OH}^{*}$ in an axisymmetrick laminar diffusion flame. 27th Symposium on Combustion Proceedings. The Combustion Institute. 615-23.

3. Docquier, N., S. Belhalfaoui, F. Lacas, N. Darabiha, and C. Rolon. 2000. Experimental and numerical study of chemiluminescence in methane/air high-pressure flames for active control applications. Proc. Combust. Inst. 28:1765-74.

4. Najm, H. N., P. H. Paul, C. J. Mueller, and P. S. Wyckoff. 1998. On the adequacy of certain experimental observables as measurements of flame burning rate. Combust. Flame 113:312-32.

5. Haber L. C., U. Vandsburger, W. R. Saunders, and V. K. Khanna. 2000. An experimental examination of the relationship between chemiluminescent light emissions and heat-release rate under non-adiabatic conditions. RTO AVT Symposium on Active Control Technology for Enhanced Performance Operational Capabilities of Military Aircraft, Land Vehicles and Sea Vehicles. Braunschweig, Germany.

6. Hardalupas, Y., and A. Selbach. 2002. Imposed oscillations and non-premixed flames. Prog. Energy Combust. Sci. 28:75-104.

7. Bernier, D., S. Ducruix, F. Lacas, S. Candel, N. Robart, and T. Poinsot. 2003. Transfer function measurements in a model combustor: Application to adaptive instability control. Combust. Sci. Technol. 175(5):993-1013.

8. Hardalupas, Y., and M. Orain. 2004. Local measurements of the time-dependent heat release rate and equivalence ratio using chemiluminescent emission from a flame. Combust. Flame 139:188-207.

9. Balachandran, R., B. O. Ayoola, C. F. Kaminski, A. P. Dowling, and E. Mastorakos. 2005. Experimental investigation of the nonlinear response of turbulent premixed flames to imposed inlet velocity oscillations. Combust. Flame 143:37-55.

10. Lauer, M., M. Zellhuber, T. Sattelmayer, and C. Aul. 2011. Determination of the heat release distribution in turbulent flames by a model based correction of $\mathrm{OH}^{*}$ chemiluminescence. J. Eng. Gas Turbines Power 133.

11. Sliphorst, M., S. Groening, B. Knapp, and M. Oschwald. 2011. Combustion instability coupling mechanisms between acoustincs and $\mathrm{LO}_{x} / \mathrm{CH}_{4}$ spray flames. AIAA Paper No. 2011-327.

12. Richecoeur, F., P. Scouflaire, S. Ducruix, and S. Candel. 2006. High-frequency transverse acoustic coupling in a multiple-injector cryogenic combustor. J. Propul Power 22(4).

13. Pomeroy, B., C. Morgan, and W. Anderson. 2011. Response of a gas-centered swirl coaxial injector to transverse instabilities. AIAA Paper No. 2011-5698.

14. Pomeroy, B., N. Nugent, and W. Anderson. 2010. Measuring transverse stability combustion response at full scale frequencies in a subscale combustor. AIAA Paper No. 2010-7146. 